\title{
Some medical applications of thermomechanically treated titanium nickelide
}

\author{
E.P. Ryklina
}

Moscow Steel and Alloys Institute, Leninsky Prospect 4, Moscow 117936, Russia

\begin{abstract}
An original device and a method of its application for restoring of the function of relatively incompetent valves (both patented) are elaborated. Application of the new device allows to lower the difficulty of surgical treatment, to decrease the duration of operation and post-operative period. The long-term results of six-year long experience of its application are presented. The patients examination after 2,5-3,0 -year post-operation period shows perfect vein valve correction.

A device for stone extraction from tabular organs (patented) fabricated with titanium nickelide superelastic alloy is presented. The new suggested design is free of the drawback inherent in the previous one. The working element of the device is formed as a truncated cone or a truncated cone coaxial with the cylinder (the previous design was formed as a full cone) that prevents overstraining and residual strain accumulation during the manipulation process.

Since the first publication the design of spiral stent and its delivering system were exposed to significant changes. In spite of a number of successful operations carried out by mears of reported devices the last one is not free from some negative features. The new patented variants of the device for stent implantation also differs from the previous one by the performing of the assembly for adjusting and fixing the position of the stent. The new device comprises superelastic nitinol working elements which provide efficiency and reliability.
\end{abstract}

\section{INTRODUCTION}

Nitinol functional properties such as shape memory and superelasticity are suitable for application in special medical devices.

Special thermomechanical treatment of nitinol wire or band allows to obtain the required working temperature range, shape memory effect or superelasticity realization, high recovery stress and also required working shape in one treatment cycle. The thermomechanical treatment includes cold or warm deformation and post-deformation heating which induces the combined polygonization and strain-aging processes. The thermomechanically treated nitinol was used for manufacturing of a set of medical devices.

\section{EXPERIMENTAL / RESULTS / DISCUSSION}

\subsection{The extravascular corrector of vein valves}

The earlier reported [1] method of extravascular correction of the function of incompetent vein valves and an extravascular corrector for that purpose (both patented) last to use in the clinical practice in Russia.

The necessity to elaborate the new design of the corrector was based on presence of serious drawbacks of known devices with circular cross-section [2].

The first one concerns the necessity of significant vein mobilization while placing the corrector accompanied by patient trauma (the long-time scar-forming processes, requiring the turnstils application). 
The most essential fact is that the corrector with round cross-section significantly diminishes the vein clearance thus reducing its capacity (Fig. 1, a). Application of the corrector with elliptic cross-section allows to achieve adequate correction, to improve the venous blood back-flow because of a relatively small reduction of a vein cross-section during said correction (Fig.1, b), [3]. When the vein compression is performed with an elliptic corrector, the free edges of which are parallel to the free edges of the valve cusps so that the joining line of valve cusps divides in half the gap between free edges of the corrector, the valve cusps move closer to each other, thus restoring the normal functioning of the valve.

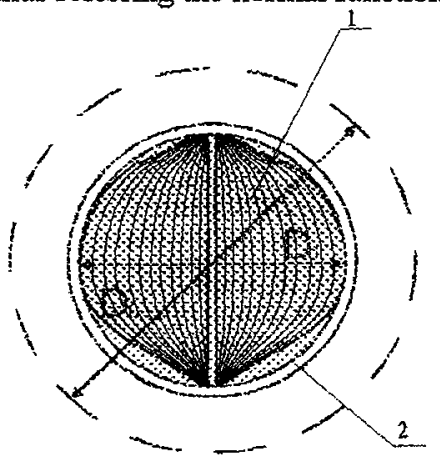

a)

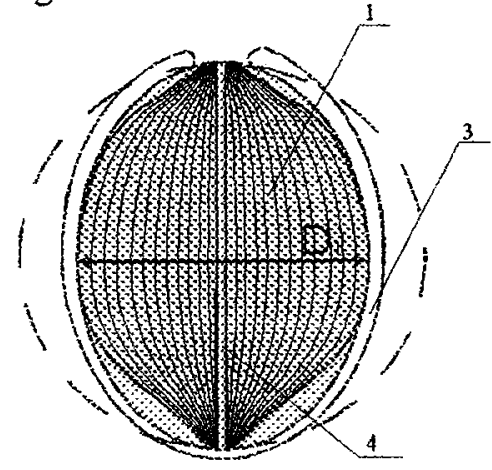

b)

1 - the valve cusps, 2 - the circular corrector, 3 - the elliptic corrector, 4 - the joining line of valve cusps; $D_{0}$ - the initial vein diameter; $D_{1}$ - the vein diameter after its correction

Figure 1: - A Schematic View Representing Changes in a Venous Cross-Section due to Vein Compression by a Circular Corrector (a) and Elliptic Corrector (b).

The elaborated corrector represents a nitinol framework with elliptic cross-section.

Variants of said corrector design are shown in Fig.2. The functioning principle of the corrector

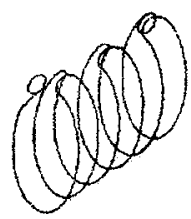

a)

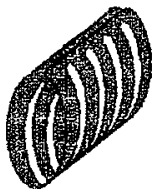

b)

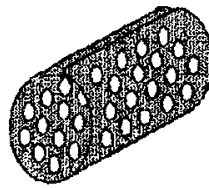

c)

Figure 2: Variants of the Corrector Design

is based on the shape memory effect, which permits to submit the device to significant pseudoplastic deformation (in our case unfolding the corrector apexes in the ice bath). Being brought to the place of implantation the corrector restores its initial shape of elliptic cylinder, surrounding the vein and changing its section in the necessary place.

All oversaid problems connected with using devices with the circular cross-section are successfully solved by using the new corrector.

The corrector was applied in the surgical treatment of 42 patients, aged from 20 to 72 , suffering from varicose disease of low extremities with the femoral vein incompetence of II-III stage. The patients were operated on at the department of General Surgery of Russian State Medical University (Clinical Hospital No 4, Moscow). Under test phlebography in $85 \%$ cases the adequate correction was achieved. In other 
cases (pathology of valve cusps) the patients showed partial correction of main vein valves. The patients examination after 1-1,5 -year post-operation period shows perfect vein value correction.

\subsection{Devices for foreign bodies extraction from hollow organs}

Concrements of bile and urine passages lead to the development of inflammations which are as a rule accompanied by considerable pains and lead to serious complications. The most widespread and nontraumatic method for such patients treatment concludes in evacuation of concrements by means of different types of removal sets. Devices known are characterized by common negative features: most of them are made of stainless steel that limits their field of application and durability.

Besides this the types of removal sets known don't allow to solve all problems connected with difficulties of stones reaching and catching. It can take place, for example, in very narrow and pathologically winding ducts, where the space is too small for opening removal sets known. The design of the working element does not provide the reliable seizure of the stone and in the case of its slipping out rather complex manipulations are necessary for its repeated catch and withdrawal which may be accompanied with an additional injury of the hollow organ. Another problem concerns the possibility of stone release in the case of its wrong seizure.

The new patented device for extraction of foreign bodies from tubular organs «Trawl» fabricated of NiTi shape memory alloy wire is free of oversaid drawbacks and allows to solve these problems [4].

The working element of the device «Trawl» is formed by two branches one of which is made in the form of a spiral helical framework; another one - straight - is placed inside the spiral branch. The straight branch plays the role of the power pivot of the whole construction and provides the required rigidity of the working element during its drawing out of the catheter and other manipulations. The helical branch provides the required rigidity during the capture, pulling, evacuation and other manipulations with the concrement. Both branches are conducted by means of the original manipulator which allows to conduct said

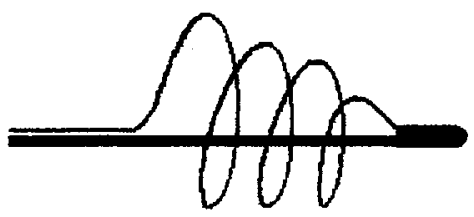
branches separately and simultaneousty. The stone extraction is carried out by means of separate conducting of wire elements. If necessary calculus release can be provided with simplicity by straightening the helical branch, that reduces patient trauma.

Figure 3: The Working Element of the New Device «Trawl» (one of the variants)

The new suggested design is free of the drawback inherent in the previous one. The working element of the device is formed as a truncated cone (Fig.3) or a truncated cone coaxial with the cylinder (the previouse design was formed as a full cone) that prevents overstraining and residual strain accumulation during the manipulation process.

\subsection{The device for spiral stent implantation}

Progressive vessel diseases can lead to the gangrene development and consequently to the extremities amputation. Atherosclerotic pathologies of coronary vessels lead to the ischemic disease of the heart and myocardial infraction and in number of cases to the disability or death (leading cause of death in USA is a coronary diseasc).

Radical surgical intervention is accompanied by significant patient trauma and can not be often realized because of the bad condition and risk. Another way concludes in non-surgical method of such patients treatment - implantation into the vessel or hollow organ the stent which corrects its clearance.

Our studies in the field of endosurgery in collaboration with physicians of the National Research Center of Surgery of the Russian Academy of Medical Sciences are carried out from 1983 [5]. As a result it laid the foundation of the new direction of practical X-ray surgery - X-ray nitinol stenting of vessels and hollow organs. 
$\mathrm{X}$-ray nitinol stent (in special literature known as Rabkin stent) represents a spiral nitinol framework selfexpandable under human body temperature.

The stent is transported to the defeated zone by means of an original delivering device which provides quick and precise implantation into exactly the necessary place.

Up to now surgeons have a great variety of different metallic stents for different indications [6].

Since the first publication the design of spiral stent and its delivering system were exposed to significant changes.

In spite of a number of successful operations carried out by means of reported devices the last one is not free from some negative features. So while twisting the stent before implantation and untwisting it in the place of implantation it is not convenient enough to stop it in intermediate position.

The new patented variants of the device for stent implantation also differ from the previous one by the performance of the assembly for adjusting and fixing the position of the stent. The new device comprises superelastic nitinol working elements which provide efficiency and reliability.

\section{CONCLUSIONS}

1. New patented device for calculus extraction from tubular organs «Trawl» allows to solve problems connected with difficulties of calculus extraction in cases of narrow pathologically winding ducts, to provide the reliable seizure of the stone and to simplify the procedure of calculus release in the case of its wrong seizure and to decrease the risk the hollow organ injury. Besides this the elaboration of the new device contributes to arsenals of surgical instruments.

2. The application of the nitinol extravasal corrector of vein valves allows to reduce the degree of venous hypertension and possibility of the development of further pathological changes of venous system. The social effect shows reducing patient trauma, shortening procedure, hospitalization and recovery times and lowering overall treatment costs.

3. The new patented variants of the device for spiral stent implantation allow to simplify as much as possible the manipulation procedure of the loading of the stent into the delivering device and to provide the maximum comfort conditions to the surgeon when manipulating during the operation.

\section{Acknoledgments}

This work has been carried out under partial support of the Russian Foundation for Basic Research grant No 96-15-98215 and of the grant of Moscow State Technical University (Ministry of Education of Russian Federation)

\section{References}

1. S.D.Prokoshkin, I.Yu. Khmelevskaya, E.P. Ryklina et al., in Proceedings of First European Conference on Shape Memory and Superelastic Technologies, Belgium, 1999, p.2.28.

2. Kuo-Hua Zhang, Fa-Qi Liang, 1993. Abstracts, $35^{\text {th }}$ World Congress of International Society of surgery, Hong Kong, p. 439.

3. S.D. Prokoshrin, A.C. Butckevitch, A.P. Chadaev et al., in Proceeding of the Third International Conference on Intelligent Materials and Third European Conference on Smart Structures and Material.: Lyon, 1996. p. 986.

4. E.P.Ryklina, T.V. Morozova, I.Yu. Khmelevskaya, et al, in Proceedings, $2^{\text {nd }}$ International Conference on Shape Memory and Superelastic Technologies: Engineering and Biomedical Applications. Pacific Grove, California, USA, 1997, p.539.

5. I.Kh. Rabkin, V.G. Germashev, in Abstracts of All-Union Symposium «New Technologies in X-ray surgery», Moscow, 1989, p. 14.

6. Handbook of Coronary Stents. Serruys P.W., Editor-in-Chief Rotterdam Thoraxcentre, Interventional Cardiology Group, 1997. 163 p. 